\title{
Fourier Approximation for Integral Equations on the Real Line
}

\author{
S. M. Hashemiparast and H. Fallahgoul \\ Department of Mathematics, Faculty of Science, K. N. Toosi University of Technology, \\ P.O. Box 16765-165, Tehran 19697, Iran \\ Correspondence should be addressed to S. M. Hashemiparast, hashemiparast@kntu.ac.ir
}

Received 8 February 2009; Accepted 22 May 2009

Recommended by Francesco Pellicano

Based on Guass quadradure method a class of integral equations having unknown periodic solution on the real line is investigated, by using Fourier series expansion for the solution of the integral equation and applying a process for changing the interval to the finite interval $(-1 ; 1)$, the Chebychev weights become appropriate and examples indicate the high accuracy and very good approximation to the solution of the integral.

Copyright (C) 2009 S. M. Hashemiparast and H. Fallahgoul. This is an open access article distributed under the Creative Commons Attribution License, which permits unrestricted use, distribution, and reproduction in any medium, provided the original work is properly cited.

\section{Introduction}

Many numerical methods for approximating the solution of integral equations are based on Galerkin and Collocation methods (see [1,2]). Some modifications in these methods such as iterated Galerkin and iterated Collocation methods give more accurate approximations to the solution [3], some others have extended the technics to Petrov-Galerkin and Hammersteion equations degenerate kernel methods (see [4-7]), in some of recent methods further improvement in some steps is not possible $[3,6]$, the computation of sufficiently accurate solution may require the use of much partition of the domain and may thus involve the solution of a correspondingly large linear system. On the other hand, it is well known that the coefficient matrix of an integral equation is dense, and it requires heavy work to generate the coefficient matrix if the partition is much finer (see $[8,9])$. To improve the difficulties in this methods, improved iterated Galerkin methods are recommended (see [10]), other improvement with less additional computational efforts for each step of iteration are introduced and shows that every step of iteration the coefficient matrix of the linear system to be inverted remains the same as that in the original approximation method (see [11]), the additional order of convergence for every step of iteration is $h^{4 r}$, which is less than $h^{2 r}$, in Galerkin, Collocation and degenerate kernel methods, where $h$ is the norm of the partition and $r$ is order of the piecewise polynomials used in the approximation. Less works consider 
the integral equations on the real line (see $[12,13]$ ), integral equations with singularity (see $[14,15]$ ), integral equations with periodic solutions (see [16]).

In this paper we consider the approximate solutions of Fredholm integral equations on the real line having periodic solutions.

We consider the following Fredholm integral equations of second kind with periodic solution on the real line

$$
f(x)=g(x)+\lambda \int_{\mathbb{R}} k(x, t) f(t) d t
$$

the main part of the first section considers the several admissibility results and existence principle on real line, however before proceeding to other sections we recall some facts about periodic functions, we discuss the general approach that will be taken to obtain the various existence principles [16].

\section{Fredholm Integral Equation on the Real Line}

Let $0<T<1$ and suppose $I$ is an interval of $\mathbb{R}$ that contains at least one compact subinterval of length $T$, which we denote by $I_{T}$. We define $A_{T}(I)$ to be the subspace of $B C(I)$ (bounded continuous functions on $I$ with values in $\mathbb{R})$ consisting of all $T$-periodic mappings, that is, if $f \in A_{T}(I)$, then $\mathrm{f}$ is continuous on $I$ and $f(t+T)=f(t)$, for all $\mathrm{t}$ such that $t+T \in I$. The norm on $A_{T}(I)$ is the same as the norm $\|\cdot\|_{0}$ on $B C(I)$, and it is clear that for $f \in A_{T}(I)$ we have

$$
\|f\|_{0}=\sup _{t \in I}|y(t)|=\sup _{t \in I_{T}}|y(t)|
$$

We consider Fredholm integral equations over the real line $\mathbb{R}$

$$
f(x)=g(x)+\lambda \int_{\mathbb{R}} k(x, t) f(t) d t
$$

that $k(x, t)$ is separable

$$
\begin{aligned}
k(x, t) & =\sum_{i=1}^{n} \gamma_{i}(x) \omega_{i}(t) \\
\omega_{i}(t) & =\frac{P_{i}\left(t^{2}\right)}{Q_{i}\left(t^{2}\right)}, \quad i=1,2 \ldots, n_{i}
\end{aligned}
$$

where

$$
Q_{i}(t)=\prod_{k=1}^{n_{i}}\left(t+b_{k}^{2}\right), \quad 0<b_{1} \leq b_{2} \leq \cdots \leq b_{n_{i}},
$$

and $P_{i}(t)$ is a polynomial of degree at most $m_{i}<n_{i}$, which is nonnegative on the half line $[0,+\infty)$, and $\gamma_{i}(x)$ is an arbitrary function. 
Problem (2.2) can be simplified by first obtaining the partial fraction decomposition of (2.4) in the form

$$
\omega_{i}(t)=\sum_{j=1}^{m_{i}} \sum_{v_{i}=1}^{r_{j}} \frac{c_{j v_{i}}}{\left(t^{2}+b_{j}^{2}\right)^{2}}
$$

where the sum is over all pairs of conjugate complex poles $\pm i b_{j}$, of $Q\left(t^{2}\right)$, with corresponding multiplicities $r_{j}, j=1, \ldots, m_{i}$. Here, $\sum_{j=1}^{m_{i}} r_{j}=n$.

Thus, without loss of generality we can consider only weights of the form

$$
\omega_{v_{i}}(t)=\omega_{v_{i}}(t ; b)=\frac{1}{\left(t^{2}+b^{2}\right)^{v_{i}}}, \quad v_{i} \geq 1
$$

In (2.2) the function $f(x)$, is $2 \pi$-periodic:

$$
\begin{aligned}
f(x) & =g(x)+\lambda \int_{\mathbb{R}} \sum_{i=1}^{n} \gamma_{i}(x) \omega_{v_{i}}(t) f(t) d t \\
& =g(x)+\lambda \sum_{i=1}^{n} \gamma_{i}(x) \int_{\mathbb{R}} \omega_{v_{i}}(t) f(t) d t \\
& =g(x)+\lambda \sum_{i=1}^{n} \gamma_{i}(x) \int_{\mathbb{R}} \frac{f(t)}{\left(t^{2}+b^{2}\right)_{i}^{v}} d t .
\end{aligned}
$$

Let

$$
I_{v_{i}}(f)=I_{v_{i}}(f ; b)=\int_{\mathbb{R}} f(t) \frac{d t}{\left(t^{2}+b^{2}\right)^{v_{i}}}, \quad b>0, v_{i} \geq 1
$$

\section{Reduction of the Integral to a Finite Interval}

Milovanovic and et al. [17] show how to reduce the integral (2.9) to an integral on the finite interval. For this purpose we need the sum of the following series:

$$
W_{v_{i}}(t)=W_{v_{i}}(t ; b)=\sum_{k=-\infty}^{\infty} w_{v_{i}}(2 k \pi+\tau)=\sum_{k=-\infty}^{\infty} \frac{1}{\left[(2 k \pi+\tau)^{2}+b^{2}\right]^{v_{i}}}
$$

Since (cf. [9])

$$
\sum_{k=-\infty}^{\infty} \frac{1}{(k+\alpha)^{2}+\beta^{2}}=\frac{\pi}{\beta} \frac{\sinh 2 \pi \beta}{\cosh 2 \pi \beta-\cos 2 \pi \alpha}
$$


in the simplest, but the most important case $v_{i}=1$, for $2 \pi \alpha=\tau$ and $2 \pi \beta=b$, we obtain

$$
W_{1}(\tau)=W_{1}(t ; b)=\frac{\sinh b}{2 b} \frac{1}{\cosh b-\cos \tau}
$$

We bring a lemma and two theorems in the following which have been proved in [17].

Lemma 3.1. Let $w_{v_{i}}$ be given by $(2.7), \zeta^{ \pm}=-(\tau \pm i b) / 2 \pi$ and $\zeta=-\zeta^{+}$. Then

$$
W_{v_{i}}(\tau)=-\frac{(2 \pi)^{1-2 v_{i}}}{2\left(v_{i}-1\right) !}\left\{\lim _{z \rightarrow \zeta^{+}} \frac{d^{v_{i}-1}}{d z^{v_{i}-1}}\left[\frac{\cot \pi z}{(z+\zeta)^{v_{i}}}\right]+\lim _{z \rightarrow \zeta^{-}} \frac{d^{v_{i}-1}}{d z^{v_{i}-1}}\left[\frac{\cot \pi z}{(z+\zeta)^{v_{i}}}\right]\right\}
$$

The proof of this result can be done by an integration of the function $z \mapsto g(z)=$ $\pi \cot (\pi z) w_{v_{i}}(2 \pi z+\tau)$ over the rectangular contour $C_{N}$ with vertices at the points $(N+$ $(1 / 2))\left( \pm 1 \pm i\right.$, ) where $N \in \mathbb{N}$ is such that the poles $\zeta^{ \pm}$of the function $g$ are inside of $C_{N}$. Then, taking $N \rightarrow+\infty$, the corresponding integral over $C_{N}$ tends to zero, because $w_{v_{i}}(z)=\mathbf{O}\left(1 / z^{2 v_{i}}\right)$ when $z \rightarrow \infty$. Then by Cauchy's residue theorem, we get

$$
W_{v_{i}}(\tau)=\sum_{k=-\infty}^{\infty} w_{v_{i}}(2 k \pi+\tau)=-\left(\operatorname{Res} g(z)_{z=\zeta^{+}}+\operatorname{Res} g(z)_{z=\zeta^{-}}\right)
$$

For $v_{i}=1$, (3.4) reduce to (3.3). When

$$
W_{v_{i}}(\tau)=\frac{b \cosh b-\sinh b}{4 b^{3}} \cdot \frac{\cos \tau+a}{(\cosh b-\cos \tau)^{2}}
$$

where

$$
a=\frac{\sinh 2 b-2 b}{2 b \cosh b-2 \sinh b} .
$$

We can suspect the following form of our sum:

$$
W_{v_{i}}=\frac{p_{v_{i}}(\cos \tau)}{(\cosh b-\cos \tau)^{2}}
$$

where $p_{v_{i}}(x)$, is an algebraic polynomial. Indeed, we can prove the following result.

Theorem 3.2. Let $x=\cos \tau$ and $c=\cosh b$. Then

$$
W_{v_{i}}(\tau)=W_{v_{i}}(\tau ; b)=\frac{p_{v_{i}}(x)}{(c-x)^{v_{i}}}, \quad v_{i}=1,2 \ldots, i=1,2 \ldots,
$$


where $p_{v_{i}}(x)=p_{v_{i}}(x ; b)$ is a nonnegative polynomial on $[-1,1]$ of degree $v_{i}-1$. These polynomials satisfy the recurrence relation

$$
p_{v_{i}+1}(x)=\frac{1}{2 b v_{i}}\left\{v_{i} \sqrt{c^{2}-1} p_{v_{i}}-(c-x) \frac{\partial p_{v_{i}}(x)}{\partial b}\right\}
$$

where

$$
p_{1}(x)=\frac{\sqrt{c^{2}-1}}{2 b}
$$

Proof. We start with (3.3) written in the form $(c-x) W_{1}(\tau)=p_{1}(x)$, where

$$
p_{1}(x)=\frac{\sinh b}{2 b}=\frac{\sqrt{c^{2}-1}}{2 b}, \quad x=\cos \tau, c=\cosh b .
$$

Thus, the formula (3.9) is true for $v=1$.

Suppose that (3.9) holds for some $(v \geq 1)$. Then, differentiating

$$
(c-x)^{v} W_{v}(\tau)=p_{v}(x)
$$

with respect to $b$, we get

$$
v(c-x)^{v-1} \frac{d c}{d b} W_{v}(\tau)+(c-x)^{v}=\frac{\partial p_{v}(x)}{\partial b},
$$

that is,

$$
(c-x)^{v+1} W_{v+1}(\tau)=\frac{1}{2 b v}\left\{v \sqrt{\left(c^{2}-1\right)} p_{v}(x)-(c-x) \frac{\partial p_{v}(x)}{\partial b}\right\}=p_{v+1}(x) .
$$

Thus, the result is proved.

We are ready now to give a transformation of the integral (2.9) to one on a finite interval. Putting $t=2 k \pi+\tau$ and using the periodicity of the function $f$,

$$
f(t)=f(2 k \pi+\tau)=f(\tau)
$$


we have

$$
\begin{aligned}
I_{v_{i}}(f)=I_{v_{i}}(f ; b) & =\sum_{k=-\infty}^{\infty} \int_{(2 k-1) \pi}^{(2 k+1) \pi} f(t) w_{v_{i}}(t) d t \\
& =\sum_{k=-\infty}^{\infty} \int_{-\pi}^{+\pi} f(\tau) w_{v_{i}}(2 k \pi+\tau) d \tau \\
& =\int_{-\pi}^{\pi} f(\tau)\left(\sum_{k=-\infty}^{\infty} w_{v_{i}}(2 k \pi+\tau)\right) d \tau,
\end{aligned}
$$

because of the uniform convergence of the series (3.1). Thus,

$$
I_{v_{i}}(f)=\int_{-\pi}^{+\pi} f(\tau) w_{v_{i}}(\tau) d \tau
$$

where $w_{v_{i}}(\tau)$ is defined by (3.1) and given by Theorem (3.2). We see that $w_{v_{i}}(-\tau)=w_{v_{i}}(\tau)$, that is, $w_{v_{i}}$ is an even weight function.

Because of the last property of the weight function, we have

$$
\begin{aligned}
I_{v_{i}}(f)=I_{v_{i}}(f ; b) & =\int_{-\pi}^{0} f(\tau) w_{v_{i}}(\tau) d \tau+\int_{0}^{\pi} f(\tau) w_{v_{i}}(\tau) d \tau \\
& =\int_{0}^{\pi}(f(\tau)+f(-\tau)) w_{v_{i}}(\tau) d \tau .
\end{aligned}
$$

Changing the variables $\cos \tau=x$ and putting

$$
f(\tau)+f(-\tau)=F(\cos \tau)
$$

we get the following result.

Theorem 3.3. The integral (2.9) can be transformed to the form

$$
I_{v_{i}}(f)=I_{v_{i}}(f ; b)=\int_{-1}^{1} F(x) \frac{p_{v_{i}}(x)}{(c-x)^{v_{i}}} \cdot \frac{d x}{\sqrt{1-x^{2}}}
$$

where $c=\cosh b, p_{v_{i}}(x)$ is a polynomial determined by the recurrence relation Theorem 3.2, and $F$ is defined by (3.20).

\section{Gaussian Type Formula for Chebychev Weights}

In order to evaluate the integral (3.20) it would seem more natural and simpler to apply the Gauss-Chebychev quadrature formula, that is, taking $x \mapsto \phi(x)=F(x) p_{v_{i}}(x) /(c-x)^{v_{i}}, c>1$, as in integrating function with respect to the Chebychev weight $(\mathrm{Chw}) v_{0}(x)=\left(1-x^{2}\right)^{-1 / 2}$. 
In this case, when for some $r \geq 1$ the function $F$ satisfied the condition

$$
\int_{-1}^{1} F^{(r)}(x)\left(\sqrt{1-x^{2}}\right)^{r-1} d x<+\infty
$$

the error $\mathbf{R}_{n}(\phi)_{v_{0}}$ of the $n$-point Gauss-Chebychev quadrature can be estimated as follow:

$$
\left|\mathbf{R}_{n}(\phi)_{v_{0}}\right| \leq \frac{A}{n^{r}} \int_{-1}^{1}\left|\frac{d^{r}}{d x^{r}}\left[\frac{F(x) p_{v_{i}}(x)}{(c-x)^{v_{i}}}\right]\right|\left(1-x^{2}\right)^{(r-1) / 2} d x
$$

where $A>0$ is a constant independent on $\phi$ and $n$. Hence, when $c>1$, is very close to 1 , even if the integrand is bounded, it gives a very large bound.

Thus, for evaluating the integral (3.20) it is more convenient to construct the Gaussian quadratures

$$
\int_{-1}^{1} \phi(x) d \lambda_{v_{i}}(x)=\frac{\pi}{n} \sum_{k=1}^{n} \phi\left(x_{i}\right)+\mathbf{R}_{n}(\phi)_{v_{0}{ }^{\prime}} \quad\left(\mathbf{R}_{n}\left(p_{2 n-1}\right)_{\nu_{0}}\right) \equiv 0,
$$

for the measure

$$
d \lambda_{v_{i}}(x)=\frac{d x}{\sqrt{1-x^{2}}}
$$

where the function $\phi$ includes the algebraic polynomial $p_{v_{i}}(x)$, that is, $\phi(x)=F(x) p_{v_{i}}(x) /$ $(c-x)^{v_{i}}$. Here, $p_{2 n-1}$ denotes the set of all polynomials of degree at most $2 n-1$ and $x_{i}$ is the root of Chebychev polynomials.

\section{Solving Integral Equation}

Let this integral equation

$$
f(x)=g(x)+\lambda \int_{R} k(x, t) f(t) d t
$$

which $f(t)$ is $2 \pi$-periodic, $k(x, t), \mathrm{BE}$ separable, that this integral equation is simplified according to Sections 2 and 3:

$$
f(x)=g(x)+\lambda \sum_{i=1}^{n} \gamma_{i}(x) \int_{-1}^{1} F(t) \cdot \frac{p_{v_{i}}(t)}{(c-x)^{v_{i}}} \cdot \frac{d t}{\sqrt{1+t^{2}}} d t
$$

where $f(\tau)+f(-\tau)=F(\cos x)=F(x), c=\cosh b$ and $p_{v_{i}}(x)$ is obtained from Theorem 3.2. 
For getting unknown function $f(x)$ in relation (5.1) which, $f(x)$ is $2 \pi$-periodic. We have the following Fourier expansion of $f(x)$ be $2 \pi$-periodic:

$$
f(x) \simeq \frac{a_{0}}{2}+\sum_{k=1}^{m}\left(a_{k} \cos k x+b_{k} \sin k x\right)
$$

indeed

$$
F(\cos \tau)=F(x)=f(\tau)+f(-\tau)=\frac{a_{0}}{2}+2 \sum_{k=1}^{m}\left(a_{k} \cos k x\right),
$$

we will obtain $2 m+1$ unknowns coefficients $a_{0}, a_{k}$ and $b_{k}, k=1 \ldots m$, by substituting in (5.2) and using Chebychev integration the relation can be solved for these unknowns, then by substituting the Fourier coefficients $f(x)$, in (5.3) will be determined.

\section{Numerical Examples}

In this section we consider some numerical examples to illustrate the presented transformation method. All computations were done by Maple 11, and the runtime for this calculation is too short. It should be noted that the real line is the integration interval of the following examples, but the integrands are periodic functions and they have been evaluated in the period $(-1,1)$.

Example 6.1. Let us consider the following integral equation:

$$
f(x)=\cos (x)-\frac{\pi}{e} \lambda \gamma(x)+\lambda \gamma(x) \int_{\mathbb{R}} \frac{f(t)}{1+t^{2}} d t,
$$

where $\lambda$ and $\gamma(x)$ are arbitrary knowns and $f(x)$ is unknown $2 \pi$-periodic function on $\mathbb{R}$, which should be determined. Also $v_{i}$ and $n$ are equal 1 in (2.8).

Applying Gaussian quadrature with the ChW for $m=5,6,7,8,9$, we get the approximate Fourier expantion of $f(x)$ with relative error given in Table 1. The exact solution of (6.1) is $\cos (x)$, the approximated solution of $f(x)$ according to the values of $a_{0}=(0.4111077167 e-3)$, and $a_{1}=1, a_{2}=a_{3}=a_{4}=a_{5}=b_{1}=b_{2}=b_{3}=b_{4}=b_{5}=0$, is $h(x)=0.4111077167 e-3 / 2+\cos (x)$ which shows the difference of the solutions for $m=5$ equals $a_{0} / 2$, and other values of $m$ the errors are given in Table 1 , which indicates a super convergence of the solution even for small values of $m$.

Example 6.2. Consider the integral equation

$$
f(x)=\frac{\sin (x)}{1+\cos (x)}+\lambda \gamma(x) \int_{\mathbb{R}} \frac{f(t)}{1+t^{2}} d t
$$

where $\lambda$ and $\gamma(x)$ are arbitrary and $f(x)$ is $2 \pi$-periodic function on $\mathbb{R}$, also $v_{i}$ and $m$ are equal 1 in (2.8). 
Table 1

\begin{tabular}{lc}
\hline$m$ & $\|f-h\|_{\infty}$ \\
\hline 5 & $2.055538584 \times 10^{-4}$ \\
6 & $2.781671562 \times 10^{-5}$ \\
7 & $1.416284140 \times 10^{-6}$ \\
8 & $5.089671730 \times 10^{-7}$ \\
9 & $6.872427665 \times 10^{-8}$ \\
\hline
\end{tabular}

Table 2

\begin{tabular}{lc}
\hline$m$ & $\|f-h\|_{\infty}$ \\
\hline 5 & $7.26100 \times 10^{-7}$ \\
6 & $4.99000 \times 10^{-8}$ \\
8 & 0 \\
\hline
\end{tabular}

Applying Gaussian quadrature with the Chw, for $m=5,6,8$.

The exact and approximated solutions are considered to illustrate in Table 2 with comparison is exact solution the $f(x)=\sin (x) /(1+\cos (x))$, for $m=8$ the error is almost zero.

Example 6.3. Consider the following integral equation:

$$
f(x)=\sin (x)+\lambda \gamma(x) \int_{\mathbb{R}} \frac{f(t)}{\left(1+t^{2}\right)^{2}} d t
$$

where $\lambda$ is arbitrary amount and $\gamma(x)$ is any function of $x$. If $v_{i}=2$ and $n=1$, by getting (Chw) quadrature we will obtain the approximations function for $f(x)$. The exact solution is $f(x)=\sin (x)$, if $m=5$ then $b_{1}=1$ and $a_{i}=b_{j}=0$, for $i=1, \ldots, 5$, and $, j=2, \ldots, 5$. So the approximation and exact solution almost equal, or $f(x)=h(x)$. The approximation solution is almost exact.

\section{Conclusion}

A Fourier series approximation for the integral equations having periodic solution are considered in the real line, the procedure produced almost an exact approximation for the solution of the integral even by taking few number of the elements in the Fourier series expansion of the solution, the numerical examples indicate the high accuracy of the approximation.

\section{References}

[1] K. E. Atkinson, The Numerical Solution of Integral Equations of the Second Kind, vol. 4 of Cambridge Monographs on Applied and Computational Mathematics, Cambridge University Press, Cambridge, UK, 1997.

[2] R. Kress, Linear Integral Equations, vol. 82 of Applied Mathematical Sciences, Springer, Berlin, Germany, 1989. 
[3] I. H. Sloan, "Four variants of the Galerkin method for integral equations of the second kind," IMA Journal of Numerical Analysis, vol. 4, no. 1, pp. 9-17, 1984.

[4] Z. Chen and Y. Xu, "The Petrov-Galerkin and iterated Petrov-Galerkin methods for second-kind integral equations," SIAM Journal on Numerical Analysis, vol. 35, no. 1, pp. 406-434, 1998.

[5] Z. Chen, Y. Xu, and J. Zhao, "The discrete Petrov-Galerkin method for weakly singular integral equations," Journal of Integral Equations and Applications, vol. 11, no. 1, pp. 1-35, 1999.

[6] I. G. Graham, S. Joe, and I. H. Sloan, "Iterated Galerkin versus iterated collocation for integral equations of the second kind," IMA Journal of Numerical Analysis, vol. 5, no. 3, pp. 355-369, 1985.

[7] H. Kaneko and Y. Xu, "Superconvergence of the iterated Galerkin methods for Hammerstein equations," SIAM Journal on Numerical Analysis, vol. 33, no. 3, pp. 1048-1064, 1996.

[8] Z. Chen, C. A. Micchelli, and Y. Xu, "A multilevel method for solving operator equations," Journal of Mathematical Analysis and Applications, vol. 262, no. 2, pp. 688-699, 2001.

[9] Z. Chen, C. A. Micchelli, and Y. Xu, "Fast collocation methods for second kind integral equations," SIAM Journal on Numerical Analysis, vol. 40, no. 1, pp. 344-375, 2002.

[10] D. Porter and D. S. G. Stirling, "The reiterated Galerkin method," IMA Journal of Numerical Analysis, vol. 13, no. 1, pp. 125-139, 1993.

[11] G. Long and G. Nelakanti, "Iteration methods for Fredholm integral equations of the second kind," Computers \& Mathematics with Applications, vol. 53, no. 6, pp. 886-894, 2007.

[12] C. Frammartino, C. Laurita, and G. Mastroianni, "On the numerical solution of Fredholm integral equations on unbounded intervals," Journal of Computational and Applied Mathematics, vol. 158, no. 2, pp. 355-378, 2003.

[13] M. C. De Bonis, C. Frammartino, and G. Mastroianni, “Numerical methods for some special Fredholm integral equations on the real line," Journal of Computational and Applied Mathematics, vol. 164-165, pp. 225-243, 2004.

[14] D. O’Regan, R. P. Agarwal, and K. Perera, "Nonlinear integral equations singular in the dependent variable," Applied Mathematics Letters, vol. 20, no. 11, pp. 1137-1141, 2007.

[15] Y. Cao, M. Huang, L. Liu, and Y. Xu, "Hybrid collocation methods for Fredholm integral equations with weakly singular kernels," Applied Numerical Mathematics, vol. 57, no. 5-7, pp. 549-561, 2007.

[16] D. O'Regan and M. Meehan, "Periodic and almost periodic solutions of integral equations," Applied Mathematics and Computation, vol. 105, no. 2-3, pp. 121-136, 1999.

[17] G. Mastroianni and G. V. Milovanović, "Weighted integration of periodic functions on the real line," Applied Mathematics and Computation, vol. 128, no. 2-3, pp. 365-378, 2002. 


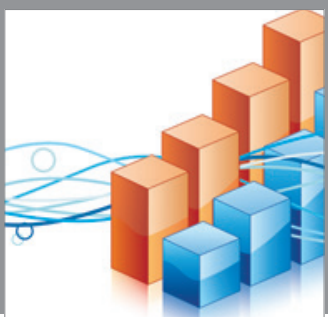

Advances in

Operations Research

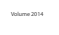

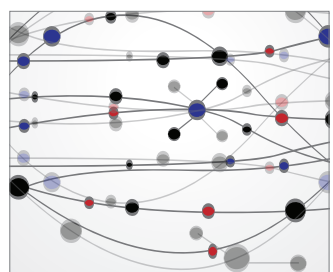

\section{The Scientific} World Journal
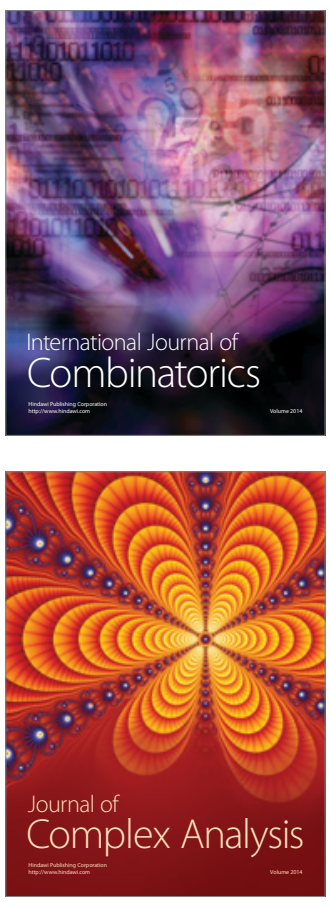

International Journal of

Mathematics and

Mathematical

Sciences
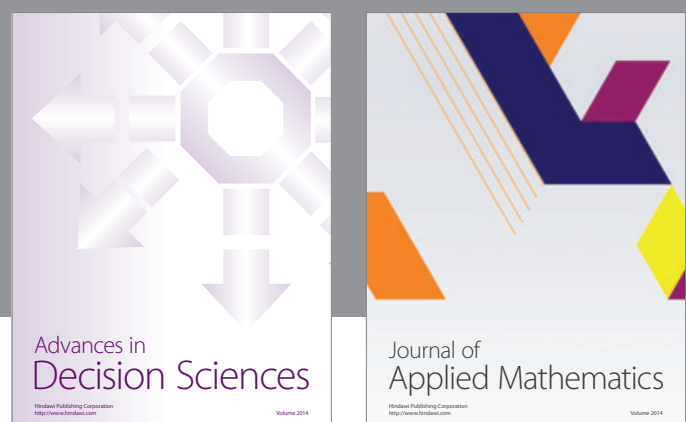

Journal of

Applied Mathematics
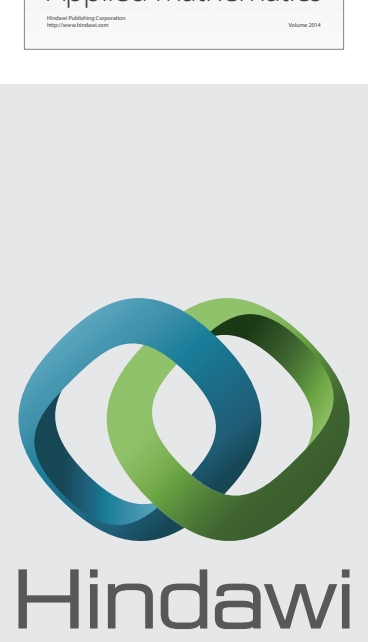

Submit your manuscripts at http://www.hindawi.com
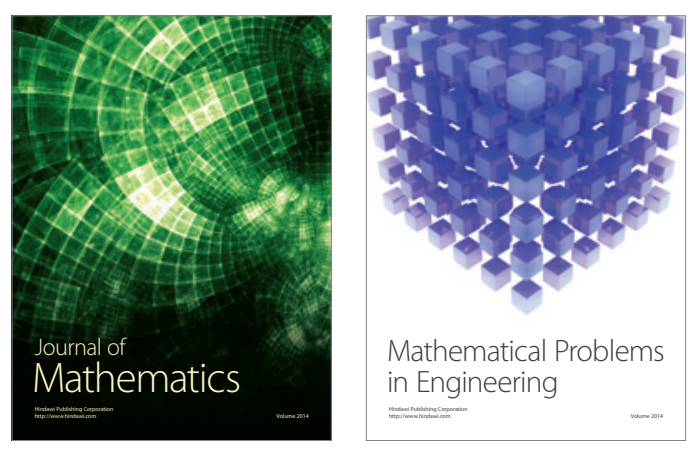

Mathematical Problems in Engineering
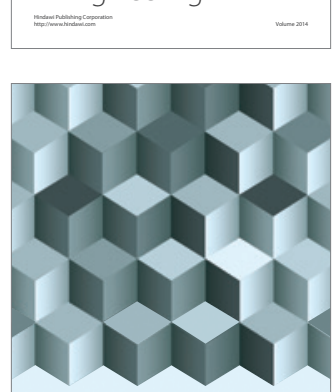

Journal of

Function Spaces
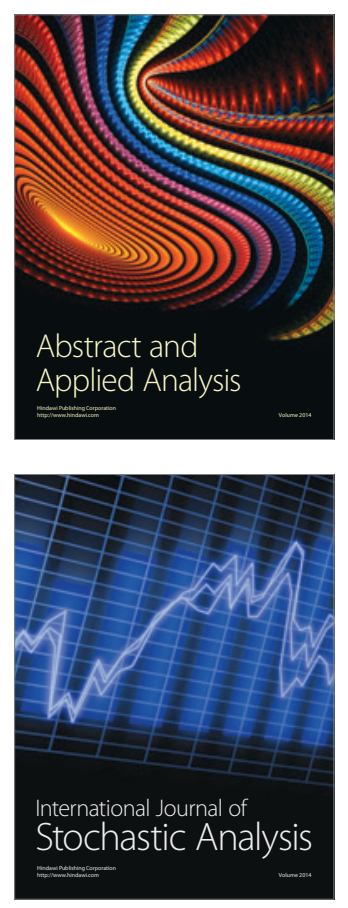

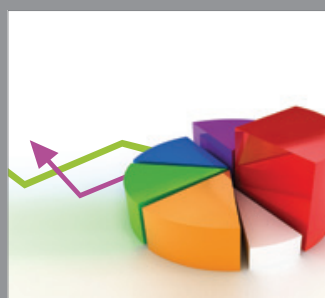

ournal of

Probability and Statistics

Promensencen
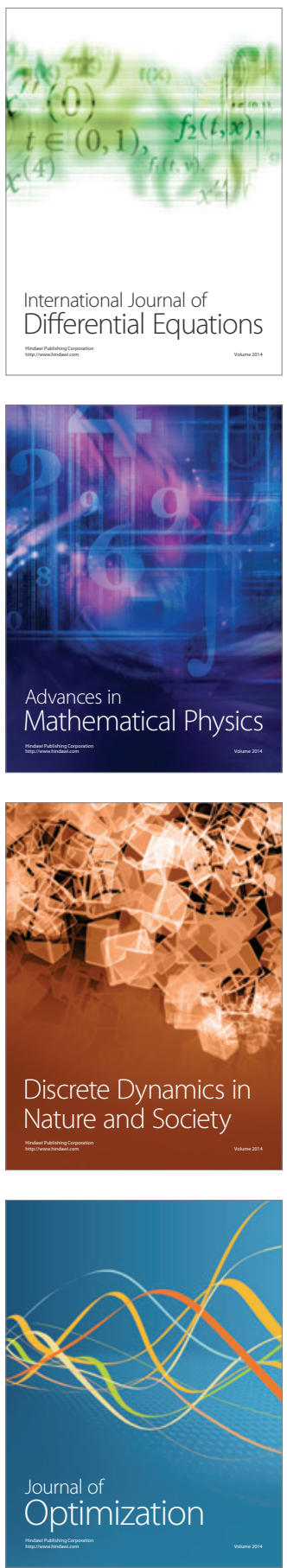